\title{
Necessary and Sufficient Conditions for Robust Stability using Modal Intervals
}

\author{
Josep Vehí ${ }^{a}$, Miguel A. Sainz ${ }^{b}$ \\ ${ }^{a}$ IIiA, Universitat de Girona, Campus Montilivi, edifici P-II, E-17071, Girona, Spain, E-mail vehi@eia.udg.es \\ ${ }^{b}$ Dept. IMA, Universitat de Girona, Girona, Spain
}

\begin{abstract}
In this paper, robustness of parametric systems is analyzed using a new approach to interval mathematics called Modal Interval Analysis. Modal Intervals are an interval extension that, instead of classic intervals, recovers some of the properties required by a numerical system. Modal Interval Analysis not only simplifies the computation of interval functions but allows semantic interpretation of their results. Necessary, sufficient and, in some cases, necessary and sufficient conditions for robust performance are presented.
\end{abstract}

\section{INTRODUCTION}

Many robust control problems can be stated as a system with a fixed model structure and nominal parameters values affected by parameter variations and a fixed controller with unknown parameters. Methods for assessing robust stability of parameter dependent linear systems often fall into two categories: those that underestimate robustness and those that overestimate robustness. Pessimistic or conservative methods underestimate robustness. These methods are usually based on some analytical result that describes sufficient (but no necessary) conditions of robust stability, for example, the small gain theorem, the circle theorem, or the Lyapunov theorem. Optimistic methods, on the other hand, overestimate robustness, often by restricting attention to a large but finite subset of $Q$. One example is Monte Carlo method.

In this paper, we will consider a class of plants with structured parametric uncertainties described by the following uncertain transfer function:

$$
G(s, q)=\frac{\alpha_{0}(q)+\alpha_{1}(q) \mathrm{s}+\cdots+\alpha_{\mathbf{m}}(q) \mathrm{s}^{\mathrm{m}}}{\beta_{0}(\boldsymbol{q})+\beta_{1}(\boldsymbol{q}) \mathrm{s}+\cdots+\beta_{\mathbf{n}}(\boldsymbol{q}) \mathrm{s}^{\mathrm{n}}}
$$

depending on a structured perturbation characterized by the parameter vector

$$
q=\left[\begin{array}{llll}
q_{1} & q_{2} & \ldots & q_{l}
\end{array}\right]^{T}
$$

where each parameter enters into the system description with polynomial dependency.

We also consider a certain configuration of the feedback system with a fixed controller $C(s, k)$, where $k$ is the design parameter vector.
The motivation of this kind of system description is that the system parameters, $\boldsymbol{q}$, can represent physical quantities that are known only to within a certain accuracy, or vary depending on operating conditions while the controller parameters, $\boldsymbol{k}$, represent degrees of freedom available to the control system designer.

Due to the physical interpretation of the uncertain parameters, each one can be considered independent from the other and their values lie between upper and lower bounds. Then, the uncertain domain can be defined as an hyperrectangle:

$$
Q=\left\{q=\left[\begin{array}{llll}
q_{1} & q_{2} & \ldots & q_{l}
\end{array}\right]^{T} \mid q_{i} \in\left[q_{i}^{-}, q_{i}^{+}\right], i=1, \ldots, l\right\}
$$

With this kind of feedback system description, several robust control problems as stability of the closed loop system, computation of the stability margins, performance analysis and robust control synthesis can be formulated. Robust performance specifications may be given as pole locations as well as $H_{\infty}$ performances expressed in the frequency domain. It is known that the solution of these problems can be reduced to the problem of checking the positivity of a set of rational functions over a given domain:

$$
f_{i}(\alpha, q, \boldsymbol{k})>\mathbf{0}, \forall \alpha \in \mathbf{A}, \forall q \in Q, \forall k \in K
$$

where $\alpha$ is the generalized frequency and $k \in \boldsymbol{K}$ may be a single point (nominal controller $\boldsymbol{k}^{0}$ ) or a certain domain in the parameter's space of the controller depending on the problem considered.

It is possible to solve these problems in an analyticalnumerical way. When the number of uncertain parameters increases, the so-called combinational explosion occurs. For practical applications, the method is recommended to be used for up to three parameters.

An obvious solution is to formulate the problem as an optimization one: first, calculate the minimum of $f_{i}(\alpha, \boldsymbol{q}, \boldsymbol{k})$ over $A, \boldsymbol{Q}$ and $\boldsymbol{K}$ and then verify positivity. This approach yields to a non convex global optimization problem of growing complexity when feedback control systems are considered.

The application of interval arithmetic in optimization problems enables a new approach: Garloff and Malan 
have applied interval arithmetic for robust control analysis and design. These approaches use standard interval algorithms in order to evaluate the range of interval functions and then, check for zero exclusion-inclusion. This straightforward translation of interval methods consist in the computation of overbounded values of the exact range of a function over a domain, then these methods can be included into the pessimistic ones. The only two answers to the question of robustness are: "yes" (the overbounded range is positive) or "maybe" (zero is included into the overbounded range). Moreover, when standard optimization algorithms are used to evaluate better ranges, the computation complexity increases, especially when these algorithms are called iteratively, as in the robust control design procedure.

In this paper, robustness of parametric systems is analyzed using a new approach to interval mathematics called Modal Interval Analysis (see, e.g., [1]). Modal Intervals are an interval extension that, instead of classic intervals, recovers some of the properties required by a numerical system. Modal Interval Analysis not only simplifies the computation of interval functions but allows semantic interpretation of their results.

Necessary, sufficient and, in some cases, necessary and sufficient conditions for robust performance will be $o b-$ tained. Also, the computational complexity will be reduced by the application of the optimallity theorems of Modal Interval Analysis. As result, the algorithms based on Modal Interval Analysis will be more efficient.

The paper is organized as follows: In the next section, some necessary concepts of Modal Interval Analysis are introduced; Section 3 presents the main results of this paper: new robustness conditions and computational simplification; it also describes the robustness checking algorithm that implements the main results.

\section{MODAl INTERVAL ANALYSiS}

Modal interval analysis [1] extends real numbers to intervals, identifying the intervals by the predicates that the real numbers fulfil, unlike classical interval analysis which identifies the intervals with the set of real numbers that they contain. In the following, some of the properties of modal intervals are stated.

Given the set of closed intervals of $\mathbf{R}, I(\mathbf{R})=\left\{[\mathbf{a}, \mathbf{b}]^{\prime} \mid\right.$ $\mathbf{a}, \mathbf{b} \in \mathbf{R}, \quad \mathbf{a} \leq \mathbf{b}\}$, and the set of logical existential and universal quantifiers $\{E, U\}$; a modal interval is defined. by a pair:

$$
X:=\left(X^{\prime}, Q X\right)
$$

where $X^{\prime} \in I(\mathbf{R})$ and $Q X \in\{E, U\} . X^{\prime}$ is called the extension and $Q X$ is the modality. The set of modal intervals will be denoted by $I^{*}(\mathbf{R})$. A modal interval $\left(\left[a_{1}, a_{2}\right]^{\prime}, E\right)$ is called existential interval or proper interval while $\left(\left[a_{2}, a_{1}\right]^{\prime}, U\right)$ is called universal interval or improper interval.

The modal quantifier $Q$ associates to every real predicate $P(.) \in \operatorname{Pred}(\mathbf{R})$ a unique interval predicate: for a variable $x \in \mathbf{R}$ and a modal interval $\left(A^{\prime}, Q A\right) \in I^{*}(\mathbf{R})$,

$$
Q\left(x,\left(A^{\prime}, Q A\right)\right):=Q A\left(x, A^{\prime}\right)
$$

The canonical notation for modal intervals is:

$$
[a, b]:=\left\{\begin{array}{lll}
\left([a, b]^{\prime}, E\right) & \text { if } & a \leq b \\
\left([b, a]^{\prime}, U\right) & \text { if } & a \geq b
\end{array}\right\}
$$

The rational operations between modal intervals are extensions of the classical interval arithmetic with the addition of the dual operator defined by:

$$
d u\left(\left[a_{1}, a_{2}\right]\right)=\left[a_{2}, a_{1}\right]
$$

The structure $\left(I^{*}(\mathbf{R}), \subseteq\right)$ is a lattice and the minimum and the maximum for a family of modal intervals $\left\{A(i) \mid A(i) \in I^{*}(\mathbf{R}), i \in I\right\}$ are called meet and join.

$$
\begin{aligned}
\text { Meet }: & \wedge(i, I) A(i)=\left[\max _{i \in I} a(i), \min _{i \in I} \overline{a(i)}\right] \\
\text { Join }: & \vee(i, I) A(i)=\left[\min _{i \in I} a(i), \max _{i \in I} \overline{a(i)}\right]
\end{aligned}
$$

The dual formulation of the modal intervals allows one to define two semantic interval functions, denoted by $f^{*}$ and $f^{* *}$ respectively, which will play a very important role in the theory because they are in close relation with the modal interval extensions and will provide meanings to the interval computations.

Definition 1 (* and **-semantic functions) If $f$ is an $\mathbf{R}^{\mathbf{n}}$ to $\mathbf{R}$ continuous function and $A \in I^{*}\left(\mathbf{R}^{\mathbf{n}}\right)$ then

$$
\begin{aligned}
f^{*}(A) & :=\vee\left(a_{p}, A_{p}^{\prime}\right) \wedge\left(a_{i}, A_{i}^{\prime}\right)\left[f\left(a_{p}, a_{i}\right), f\left(a_{p}, a_{i}\right)\right] \\
f^{* *}(A) & :=\wedge\left(a_{i}, A_{i}^{\prime}\right) \vee\left(a_{p}, A_{p}^{\prime}\right)\left[f\left(a_{p}, a_{i}\right), f\left(a_{p}, a_{i}\right)\right]
\end{aligned}
$$

where $a=\left(a_{p}, a_{i}\right)$ is the component split corresponding to $A=\left(A_{p}, A_{i}\right)$, with $A_{p}$ a subvector containing the proper components of $A$ and $A_{i}$ a subvector containing the improper components of $A$.

The two following key theorems give a meaning to the interval results $f^{*}$ and $f^{* *}$ and characterize them as modal interval extensions.

Theorem 1 ( $f^{*}$ Semantic Theorem)

Given $F: I^{*}\left(\mathbf{R}^{\mathbf{n}}\right) \rightarrow I^{*}(\mathbf{R})$, an interval extension of the continuous function $f: \mathbf{R}^{\mathbf{n}} \rightarrow \mathbf{R}$, and a modal vector $A \in I^{*}\left(\mathbf{R}^{\mathbf{n}}\right)$, then $f^{*}(A) \subseteq F(A)$ implies

$$
U\left(a_{p}, A_{p}^{\prime}\right) Q(z, F(A)) E\left(a_{i}, A_{i}^{\prime}\right) \quad z=f\left(a_{p}, a_{i}\right)
$$


A dual semantic for proper and improper modal intervals is established by the next result.

Theorem 2 ( $f^{* *}$ Semantic Theorem)

Given $F: I^{*}\left(\mathbf{R}^{\mathbf{n}}\right) \rightarrow I^{*}(\mathbf{R})$, an interval extension of the continuous function $f: \mathbf{R}^{\mathbf{n}} \rightarrow \mathbf{R}$, and a modal vector $A \in I^{*}\left(\mathbf{R}^{\mathbf{n}}\right)$, then $f^{* *}(A) \supseteq F(A)$ implies

$$
U\left(a_{i}, A_{i}^{\prime}\right) Q(z, d u(F(A))) E\left(a_{p}, A_{p}^{\prime}\right) \quad z=f\left(a_{p}, a_{i}\right)
$$

To compute the semantic extensions $f^{*}$ and $f^{* *}$ is, in general, a difficult challenge. When the continuous function $f$ is rational, it can be operationally extended to a modal rational function by using the computing program defined by the syntactical tree of the expression of the function, where the real operators are transformed into their ${ }^{*}$-or **-semantic extensions. For a class of operators, both semantic extensions are equal. In this case, there exists a modal rational function, $f R(A)$, defined by the program associated with the syntactical tree of $f$ where the real operators are transformed into their semantic extensions. However, $f R(A)$ is not interpretable. The interpretation problem for a modal rational function consists of relating it to the corresponding semantic functions, which have standard meanings defined by the semantic theorems.

There are several theorems relating the modal rational function $f R(A)$ to the modal semantic extensions $f^{*}$ and $f^{* *}$. The following ones give two *- and **-interpretable coercions.

Definition $2 A$ component $x_{i}$ of $x$ is uni-incident in a rational function $f(x)$ if it occupies only one leaf of the syntactical tree of $f$; otherwise, $x_{i}$ is multi-incident in $f(x)$.

Theorem 3 If in $f R(A)$ there are multi-incident improper components and if $A T^{*}$ is obtained from $A$ by transforming, for each if them, all incidences but one into their duals, then $f^{*}(A) \subseteq f R\left(A T^{*}\right)$.

If all components of $A$ are proper, then $A T^{*}=A$ and $f^{*}(A) \subseteq f R(A)$.

Theorem 4 If in $f R(A)$ there are multi-incident proper components and if $A T^{* *}$ is obtained from $A$ by transforming, for each of them, all incidences but one into their duals, then $f^{* *}(A) \supseteq f R\left(A T^{* *}\right)$.

If all components of $A$ are improper, then $A T^{* *}=A$ and $f^{* *}(A) \supseteq f R(A)$.

An interpretable rational interval program $f R(A)$ may nevertheless result in a loss of information far more important than that produced by numerical rounding. Then it is very important to determine criteria to characterize the rational interval functions for which the program $f R(A)$, with an ideal computation (infinite precision), has the property that $f^{*}(A)=f R(A)=f^{* *}(A)$. In this case, we say that $f R($.$) is optimal for A$.

There are several results which characterize the optimality of a modal rational function according to its monotonicity.

Definition $3 A$ continuous function $f(x, y)$, is a uniformly monotonic function of $x$ in a domain $\left(X^{\prime}, Y^{\prime}\right) \subseteq$ $\left(\mathbf{R}, \mathbf{R}^{\mathbf{m}}\right)$ if it is a monotonic function of $x$ in $X^{\prime}$ and keeps the same type of monotonicity for all $y \in Y^{\prime}$.

Definition $4 A$ continuous function $f(x, y)$, is a totally monotonic function of a multi-incident variable $x$ in a domain $\left(X^{\prime}, Y^{\prime}\right) \subseteq\left(\mathbf{R}, \mathbf{R}^{\mathbf{m}}\right)$ if it is a uniformly monotonic function of $x$ in $X^{\prime}$ and, for every incidence of $x$ considered as an independent variable, it is also uniformly monotonic.

Theorem 5 (*-partially optimal coercion) Let $A$ be an interval vector and $f R$ be defined in the domain $A^{\prime}$ and totally monotonous for a subset $B$ of multi-incident components. Let $A D T^{*}$ be the enlarged vector of $A$, such that each incidence of every multi-incident component of the subset $B$ is included in $A D T^{*}$ as an independent component, but transformed into its dual if the corresponding incidence-point has a type of monotonicity contrary to the global one; for the rest, the multi-incident improper components are transformed into their dual in every incidence except one. Then

$$
f^{*}(A) \subseteq f R\left(A D T^{*}\right)
$$

Theorem 6 (optimal coertion) Let $A$ be an interval vector and $f R$ be defined in the domain $A^{\prime}$ and totally monotonous for all its multi-incident components. Let $A D^{*}$ be the enlarged vector of $A$, such that each incidence of every multi-incident component is included in $A D^{*}$ as an independent component, but transformed into its dual if the corresponding incidence-point has a type of monotonicity contrary to the global one of the corresponding A-component. Then

$$
f^{*}(A)=f R(A D)=f^{* *}(A)
$$

\section{ROBUSTNESS ANALYSIS}

As seen in section 1, checking the robustness of a controlled system is equivalent to verifying the positiveness of the range of a set of functions. Therefore the study of robustness stability or robust performance of uncertain systems can be generalized to the study of uncertain functions and hence these functions can be labeled as stable or not.

Definition 5 An uncertain function $F_{i}(q)$ is stable over $Q$ if $F_{i}(q)>0$ for all $q \in Q$ 
Definition 6 An uncertain function $\boldsymbol{F}_{i}(\boldsymbol{q})$ is unstable over $Q$ if there exists at least one $q^{0} \in Q$ such that $\boldsymbol{F}_{i}\left(q^{0}\right)<0$

According to the definitions stated above, the semantic interpretations of $f^{*}$ and $f^{* *}$ are very closely related with the concept of robust stability [2]. *-semantics can be used for testing stability and ${ }^{* *}$-semantics can check instability. So, according to the above results obtained for rational functions, optimality implies necessary and sufficient positivity conditions. Outer approximations of $f^{*}$ will yield sufficient positivity conditions and necessary conditions will be obtained by inner approximations of $f^{* *}$.

It will also be shown that the application of the previous theorem entails faster and simpler computations.

In order to formulate the positivity conditions by using modal intervals, the uncertainty domain should be represented as a suitable set of modal intervals. An uncertain domain means that every uncertain parameter $q_{i}$ has an unknown value between $q_{i}$ and $\overline{q_{i}}$. This fact means considering every uncertain parameter as a proper or existential modal interval:

$$
q_{i}=\left(\left[\underline{q_{i}}, \overline{q_{i}}\right], E\right) \Rightarrow q_{i}=\left[\underline{q_{i}}, \overline{q_{i}}\right], \quad \underline{q_{i}} \leq \overline{q_{i}} .
$$

A straightforward translation of the semantic theorems to the interval function obtained by substituting the uncertain parameters by proper intervals gives the next theorem:

Theorem 7 Consider a function $\boldsymbol{F}_{i}(\boldsymbol{q})$ depending on an uncertain parameter vector $q$ belonging to an uncertainty domain $\boldsymbol{Q}$. The function $\boldsymbol{F}_{\boldsymbol{i}}(\boldsymbol{q})$ is stable over $\boldsymbol{Q}$ if and only if $F_{i}^{*}(q)>0$.

As *-and **-extensions are not always calculabie, some properties of modal intervals applied to rational functions must be used, in order to work with computable functions. The previous theorem is reformulated for modal rational extensions as:

Theorem 8 Let $\boldsymbol{F} \boldsymbol{R}_{i}(\boldsymbol{q})$ be a modal rational extension of $F_{i}(\boldsymbol{q}) . F_{i}(\boldsymbol{q})$ is stable over $\boldsymbol{Q}$ if and only if $\boldsymbol{F} \boldsymbol{R}_{i}(\boldsymbol{q})$ is optimal, and $F R_{i}(q)>0$.

The key problem is the study of the optimality of the rational function $\boldsymbol{F} \boldsymbol{R}_{i}(\boldsymbol{q})$. As shown in the previous section, optimality is very closely related to monotonicity.

When the function $\boldsymbol{F}_{i}(\boldsymbol{q})$ is monotonic for all or some of its variables, modal interval analysis gives conditions of optimality or, at least, interpretability of $\boldsymbol{F} \boldsymbol{R}_{i}(\boldsymbol{q})$.

If $F_{i}(q)$ is uniformly monotonic for each variable and for all its incidences, then theorem 6 can be applied to get an optimal computation. When $\boldsymbol{F}_{i}(\boldsymbol{q})$ is not totally monotonic for any variable, theorems 3 and 4 can be applied to obtain the following general conditions:
Theorem $9 \boldsymbol{F}_{\boldsymbol{i}}(\boldsymbol{q})$ is stable over $\boldsymbol{Q}$ if $\boldsymbol{F} \boldsymbol{R}_{i}\left(\boldsymbol{q} T^{*}\right)>0$

This theorem provides only a sufficient positivity condition. In order to implement a suitable stability testing algorithm, necessary conditions are needed. The following theorem gives such conditions.

Theorem 10 Consider the function $\boldsymbol{F}_{i}(\boldsymbol{q})$ described above. Let $\boldsymbol{F} \boldsymbol{R}\left(\boldsymbol{q} T^{* *}\right)=\left\{\boldsymbol{F} \boldsymbol{R}_{i}^{k}\left(\boldsymbol{q} T^{* *}\right), \quad k=1, \ldots, N\right\}$ be the set of the $N$ possible results obtained by transforming, for every multi-incident parameter, all incidences but one into its dual.

The function $F_{i}(q)$ is unstable over $Q$ if the lower bound of the interval $\left[\boldsymbol{F R _ { i } ^ { k } ( q T ^ { * * } )}, \overline{F R_{i}^{k}\left(q T^{* *}\right)}\right]$ is not positive for some $k$

In order to remark the importance of this theorem, the semantic interpretation will be analyzed according to the modality of the result.

First, suppose that a result $\boldsymbol{F} \boldsymbol{R}_{i}^{k}\left(\boldsymbol{q} T^{* *}\right)$ is proper. The meaning is: for every value of the result $c \in$ $\left[\boldsymbol{F} \boldsymbol{R}_{i}^{k}\left(\boldsymbol{q} T^{* *}\right), \overline{\left.\boldsymbol{F} \boldsymbol{R}_{i}^{k}\left(\boldsymbol{q} T^{* *}\right)\right]}\right.$, there exists a point $q \in \boldsymbol{q}$ such that $\boldsymbol{F} \boldsymbol{R}_{i}^{k}(q)=c$. In other words, there exists at least one unstable function.

Suppose now that $\boldsymbol{F} \boldsymbol{R}_{i}^{k}\left(\boldsymbol{q} T^{* *}\right)$ is improper. The semantic interpretation is: there exists a value $c \in$ $\left[\boldsymbol{F} \boldsymbol{R}_{i}^{k}\left(\boldsymbol{q} T^{* *}\right), \overline{\boldsymbol{F} \boldsymbol{R}_{i}^{k}\left(\boldsymbol{q} T^{* *}\right)}\right]$ and a point $q \in \boldsymbol{q}$ such that $\boldsymbol{F} \boldsymbol{R}_{i}^{k}(q)=$ c. If $\inf \left(\boldsymbol{F} \boldsymbol{R}_{i}^{k}\left(\boldsymbol{q} T^{* *}\right)\right) \leq 0$, then $\sup \left(\boldsymbol{F} \boldsymbol{R}_{i}^{k}\left(\boldsymbol{q} T^{* *}\right)\right) \leq 0$, and the meaning is: there exists at least one unstable function.

These conditions has been combined into an stability analysis algorithm in order to check the performance achievement of a given set $Q$. The implemented algorithm splits the uncertainty domain in the parameters space into sub-boxes according to the monotonicity.

Several examples showing the use of these algorithms as well as their use into robust control design routines can be found in [2].

\section{ACKOWLEDGEMENTS}

This work has been partially supported by the Spanish Science and Technology Council under grant TAP96-1114C03-03 and by the University and Research Commission of the Generalitat de Catalunya, ref. 1997SGR 00098.

\section{REFERENCES}

[1] E. Gardeñes, H. Mielgo, and A. Trepat, "Modal intervals: reasons and ground semantics", in: Interval Methematics 1985, Berlin: Springer, 1985.

[2] J. Vehí, Análysis i Disseny de Controladors Robustos mitjançant Intervals Modals, Ph.D. Dissertation, University of Girona, 1998. 